The findings of this small study contribute to a growing body of evidence that repeated injections with botulinum toxin type $A$ are beneficial and safe for patients with incontinence and neurogenic detrusor overactivity.

Original article Reitz A et al. (2007) Do repeat intradetrusor botulinum toxin type $\mathrm{A}$ injections yield valuable results? Clinical and urodynamic results after five injections in patients with neurogenic detrusor overactivity. Eur Urol 52: 1729-1735

\section{Retrograde urethrocystography might impair CT of hemorrhages in pelvic fracture patients}

The diagnostic work-up of patients who suffer pelvic fracture includes pelvic CT to detect arterial hemorrhage and conventional retrograde urethrography or cystography to detect lowerurinary-tract injury. The appropriate sequence in which these investigations are performed is, however, controversial. Netto and colleagues studied whether retrograde urethrocystography interferes with subsequent visualization of arterial extravasation on pelvic CT.

The authors retrospectively evaluated the data from blunt trauma patients with pelvic fracture and either a bladder or urethral rupture, seen in a single Canadian center between June 2002 and May 2006. Patients were assigned to one of two groups according to the order in which urologic tests (retrograde urethrography and cystography) and pelvic CT were performed: patients in group $1(n=23)$ underwent urologic tests first, and patients in group $2(n=26)$ underwent pelvic CT first.

Indeterminate or false-negative results for pelvic arterial extravasation on pelvic CT were significantly more frequent in group 1 than group 2 (11 of 23 patients versus 0 of 26 patients; $P<0.001)$. Furthermore, patients with indeterminate or false-negative results on CT generally had a longer mean delay before embolization than those with positive $\mathrm{CT}$ results.

The authors conclude that the ability of pelvic CT to identify pelvic artery extravasation might be impaired by previously performed retrograde urethrography or cystography, which in turn might delay treatment of pelvic artery hemorrhage.

Original article Spencer Netto FAC et al. (2007) Retrograde urethrocystography impairs computed tomography diagnosis of pelvic arterial hemorrhage in the presence of a lower urologic tract injury. J Am Coll Surg [doi:10.1016/j.jam collsurg.2007.07.025]

\section{Many sexual adverse effects of finasteride are attributable to a nocebo effect}

Finasteride, which is used in the treatment of benign prostatic hyperplasia (BPH), has been associated with a range of sexual adverse effects. The reported incidences of such effects have been lower in randomized controlled trials than in clinical practice. Mondaini et al. have investigated whether the higher incidences observed in clinical practice could be the result of a nocebo effect-an adverse effect not directly related to the specific pharmacological action of a drug.

In total, 107 men (aged 51-65 years) who had BPH but no reported sexual dysfunction completed the 1-year study period. All participants received $5 \mathrm{mg} /$ day finasteride and were randomly assigned to receive either counseling on the potential sexual adverse effects of the drug or no such counseling. Using patients' self-reports and the Male Sexual Function 4-Item questionnaire, the authors evaluated differences in the incidence of sexual dysfunction between the two study groups. At both 6 and 12 months, the overall incidence of sexual dysfunction was higher in the counseled group than in the group that had not received counseling (36.3\% vs $11.5 \%$ at 6 months; $43.6 \%$ vs $15.3 \%$ at 12 months). Individual components of overall sexual dysfunction (erectile dysfunction, decreased libido and ejaculation disorders) were also more frequently reported in the counseling group.

The authors conclude that many of the sexual adverse effects associated with finasteride can be attributed to a nocebo effect, and that this finding should influence patient management.

Original article Mondaini $\mathrm{N}$ et al. (2007) Finasteride $5 \mathrm{mg}$ and sexual side effects: how many of these are related to a nocebo phenomenon. J Sex Med 4: 1708-1712

\section{Hysterectomy is associated with an increased risk of future stress urinary incontinence}

The majority of hysterectomies - approximately $90 \%$ of procedures carried out in the US - are performed for benign indications; however, the short-term and long-term risks of lowerurinary-tract dysfunction, such as stress urinary incontinence (SUI), associated with this procedure 